frontline paediatric clinical care providers (Consultants in Paediatric Medicine, Paediatric Nurses, Pharmacists, Dieticians, Hospital Senior Management and Non-Consultant Hospital Doctors). At a series of face-to-face sessions, teams receive mentoring in QI methodology by RCPI QI Faculty, through the IHI Breakthrough Series Collaborative Model, to develop local SAFE improvement projects impacting on

- Reducing avoidable error and harm to acutely unwell children

- Improving communication between all individuals involved in a child's care

- Improving working culture for healthcare staff providing care to children

- Increasing involvement of parents, children and young people in their care.

The teaching faculty includes active patient representation through parent involvement. Participating teams are encouraged to engage with children, parents and carers to guide their improvement efforts.

Teams collect a concise monthly dataset to facilitate aggregate and comparative measures on paediatric clinical outcomes. Teams are encouraged to use this data, and to collect other necessary data to inform the outcome, process and balancing measures pertinent to the areas upon which they are focusing their improvement efforts.

The National SAFE Improvement Collaborative is in its early stages. However, at this point, experience with similar national collaborative projects indicates that QI methodology will be used effectively to generate improvements to positively impact paediatric patient safety outcomes, through site specific changes.

\section{P200 MANAGEMENT OF HENOCH SCHONLEIN PURPURA (HSP)}

${ }^{1}$ Lubna Mahmood*, ${ }^{2}$ Farah Zulfiqar, ${ }^{3}$ Saira Khalid, ${ }^{4}$ Michelle Dillon. 'St.Luke's Hospital, Kilkenny, Ireland; '2St.Luke's Hospital, Kilkenny, Ireland; ${ }^{3}$ St. Luke's Hospital, Kilkenny, Ireland; 'St. Luke's Hospital , Kilkenny, Ireland

10.1136/archdischild-2019-epa.555

Introduction HSP is a common vasculitis of childhood. It is immune mediated. Aetiology is unknown but the history often identifies a preceding throat/URTI infection.

Agreed criteria for HSP diagnosis are:

Palpable purpura (mandatory) in the presence of atleast one of the following;

Diffuse abdominal pain

Acute arthritis or arthralgia

Renal involvement (haematuria +/_ proteinuria)

Renal biopsy showing predominantly IgA deposition.

Diagnosis is usually on clinical grounds . Bloods are needed to rule out other diagnoses. All patients need BP, urine dipstick, weight and height measurements.

General supportive measures and simple analgesia is all that is required . HSP is usually self limiting. 33\% will have relapses/recurrence of symptoms. HSP accounts for $3 \%$ of all patients with end-stage renal failure .

Aim To look at management of HSP in our unit during the last six months, develop local guidelines and then re audit to monitor compliance with guidelines.

Method We carried out a retrospective observational charts review of all children presenting with HSP.
Results No standard guidelines were being followed. Followup practices differed between teams. We developed local guidelines for the management of HSP.

If the initial urinalysis is normal or only reveals microscopic haematuria, follow up involves : clinical review, BP measurement and early morning urinalysis at these recommended time intervals :

1. Weekly for the first month

2. Fortnightly from weeks 5 to 12

3. Single review at 6 and 12 months

4. Return to (1) if there is disease flare up

Referral to Paediatric Nephrologist is warranted if there is : Macroscopic haematuria more than 5 days.

Persistent microscopic haematuria beyond 12 months.

Persistent proteinuria.

Hypertension

Abnormal renal function

Nephrotic syndrome

Nephritic syndrome

We carried out retrospective charts review again in the following six months. There were 7 cases of HSP noted . 5 patients had HSP with no renal involvement and were followed according to local protocol. It was noted that three of these patients had unnecessary blood tests done including Coagulation profile. One patient was not followed up after after the initial presentation due to miscommunication but later reviewed at 3 months.

One patient has mild HSP Nephritis with persistent proteinurea and macroscopic haematuria and was referred to the nephrologist. Her symptoms resolved completely.

We will continue to audit our practise. In addition to monitoring renal status we also aim to avoid unnecessary blood tests. Streaming our followup may identify early markers of renal disease in this group of children.

\section{P201 AN AUDIT OF THE COMPLIANCE WITH A NEWLY INTRODUCED 'ELECTRONIC PATIENT DISCHARGE' SYSTEM IN THE DEPARTMENT OF PAEDIATRICS, UNIVERSITY HOSPITAL LIMERICK (UHL)}

Uzair Athar Khan*, Katie Flyn, Anne Murie Murphy, Siobhan Gallagher. UHL, Dooradoyle Limerick, Ireland

\subsection{6/archdischild-2019-epa.556}

Background Electronic discharge application is a multi-tenant based application (any hospital in the country can use it if desired). It first started in June 2017. The primary reason for introducing it was to aid communication between the hospital and General Practitioners (GP).The focus of this project was to provide a streamlined method for Non- Consultant Hospital Doctors (NCHDs) to easily generate a patient discharge summary letter in an electronic format that can be reviewed and signed off by their Consultants. This discharge summary is then available for sending to a patient's GP via electronic means or in printed hardcopy.

These requirements are based in part on the HIQA 'National Standard for Patient Discharge Summary information document. This helps in terms of ensuring that all relevant data is captured and fed back to the patients GP in a clear, concise and timely manner .In addition to supporting the primary goal, this will further support a myriad of 